\title{
The First Reported Autoimmune Hemolytic Anemic Syndrome with Systemic Lupus Erythematosus Complicated by COVID 19 Infection in Sudan: Correspondence
} \author{
Mohamed $^{2,5}$, Abdelkareem A. Ahmed ${ }^{2,9,10 *}$, Sadia Kamal Albadawi Mohamed ${ }^{11,2}$ \\ ${ }^{1}$ Department of Medicine, Faculty of Medicine, University of Bahri, Khartoum, Sudan. \\ ${ }^{2}$ Department of Clinical Medicine, Medical and Cancer Research Institute (MCRI), Nyala, Sudan \\ ${ }^{3}$ Rheumatology Research Department, Sudanese Medical Research Association, Khartoum, Sudan. \\ ${ }^{4}$ Department of Clinical Immunology, Sudan Medical Specialization Board, Khartoum, Sudan \\ ${ }^{5}$ Faculty of Medicine, Alfashir University, Alfashir, Sudan. \\ ${ }^{6}$ Surgery Research Department, Sudanese Medical Research Association, Khartoum, Sudan \\ ${ }^{7}$ Faculty of Medicine, University of Khartoum, Khartoum, Sudan. \\ ${ }^{8}$ Faculty of Medicine, Omdurman Islamic University, Khartoum, Sudan \\ ${ }^{9}$ Institute of Molecular Biology, University of Nyala, Nyala, Sudan \\ ${ }^{10}$ Department of Physiology and Biochemistry, Faculty of Veterinary Science, University of Nyala. \\ ${ }^{11}$ Department of Internal Medicine, Sudan Medical Specialization Board, Khartoum, Sudan
}

Ziryab Imad Taha Mahmoud ${ }^{1,2,3 *}$, Asaad Tageldein Idris Abdelhalim ${ }^{4}$, Mohammed Elmujtba Adam Essa ${ }^{2,5,6 *}$,Latifa M. Elsheikh7 ${ }^{7}$ Mohey Aldein Ahmed Elamin Elnour ${ }^{8}$, Ayman Sati Sati

*Corresponding author: Dr Mohammed Elmujtba Adam Essa, Department of Clinical Medicine, Medical And Cancer Research Institute, Nyala, Sudan, Email: Awadali818@yahoo.com, Tel: 00249907009389

Dr Ziryab Imad Taha, Faculty of Medicine, University of Bahri, Khartoum, Sudan

Dr Abdelkareem Abdallah Ahmed, Department of Physiology and Biochemistry, Faculty of Veterinary Science, University of Nyala, Nyala, P.0 Box: 155 Nyala, Sudan, Email: Kareemo151@gmail.com

\section{ARTICLE INFO}

Received: 业 August 02, 2020

Published: 幽 August 11, 2020

\section{ABSTRACT}

Citation: Ziryab Imad Taha Mahmoud, et al. The First Reported Autoimmune Hemolytic Anemic Syndrome with Systemic Lupus Erythematosus Complicated by COVID 19 Infection in Sudan: Correspondence. Biomed J Sci \& Tech Res 29(3)-2020. BJSTR. MS.ID.004807.

\section{Introduction}

A serious of unexplained cases with pneumonia have been reported in Wuhan, china. The disease has a very similar clinical manifestation of viral pneumonia. After multiple sequences analysis of respiratory tract samples, a novel strain of coronavirus was isolated, and has been named Coronavirus Disease 2019 (COVID19) [1]. Common symptoms include cough, fever, fatigue, and myalgia
[2]. Complications include acute cardiac injury, acute respiratory distress syndrome and secondary infections [3], however data regarding cardiovascular involvement due to COVID 19 are less described. here we describe the first case of autoimmune hemolytic anemic complicated by COVID 19 infection in Sudan. A 57-year-old Sudanese male from River Nile state presented to the Out-patient 
clinic in Almawda Hospital-Sudan in 11 April 2020 with complaint of Pyrexia of unknown origin for 5 months. The condition started at November 2019, with high grade fever, intermittent at the beginning then became continuous, relieved only by paracetamol tablets and associated with profuse sweating. There was also slight weight loss, loss of appetite, nausea, and malaise. on systemic enquiry He complained of generalized joints pain, not associated with swelling, deformity, or restriction of movement, along with generalized fatigability. However, he denied morning stiffness.

There was also severe mouth dryness, no eyes dryness, hair loss, any skin rashes or headache. There was abdominal distention, no change in bowel habits, no night sweats, cough, chest pain, hemoptysis or shortness of breath, no palpitations, dizziness or black outs, no genitourinary symptoms. He had no previous episode of prolonged fever in the past and no history of travel. On examination the patient looked ill, febrile, lethargic, pale, and severely jaundiced. He was not distressed and all of his vital parameters were normal. The cardiopulmonary examination was all normal. Air entry was equal bilaterally, there was no chest deformity, had normal vesicular breathing with no added sounds and normal first and second heart sounds with no murmur. Abdominal examination revealed huge splenomeglay up to the umbilicus, normal liver span, and negative shifting dullness. There were discrete, non-tender, firm enlarged lymph nodes in the cervical and axillary regions. Musculoskeletal examination was normal, there was no swelling, tenderness, restricted movement, or deformity along all of his body joints. There was no skin rash or any bruises, no hair thinning or area of alopecia. No lower limbs edema and peripheral pulses were intact.

After investigations, the patient discovered to have low hemoglobin $6 \mathrm{~g} / \mathrm{dl}$ (normal value 12-16 g/dl), seen by a hematologist and work-up was done including bone marrow aspirate to exclude hematological diseases. His hemoglobin continued to drop despite regular blood transfusion of 1 to 2 bind weekly. Accordingly, a hematological consultation was done for the second time; and clinical examination detected cervical and axillary lymphadenopathy, and huge splenomegaly. His blood tests showed normocytic normochromic anemia with anisocytosis, high ferritin level, increased reticulocytes count and strongly positive direct coombs test. Right axillary lymph node biopsy was taken, which was consistent with chronic lymphadenitis. A Bone marrow aspirate and trephine biopsy was taken and showed reactive bone marrow, highly suggestive of lymphoproliferative disease, accordingly a sample was submitted for flowcytometry which confirmed that the bone marrow is not infiltrated by lymphoma cells. After excluding any possibility of infections such as tuberculosis and brucellosis, and excluding of any hematological malignancies, the patient had been referred for assessment of autoimmune diseases and further work-up was done and he was prescribed hydroxychloroquine $200 \mathrm{mg}$, and methylprednisolone 80mg od IM for 3-day.

His blood tests showed elevated ESR (120 mm/hour), CRP $(102 \mathrm{mg} / \mathrm{l})$ and fine speckled pattern of the antinuclear antibodies with titer of 1/320. Accordingly, a differential diagnosis of undifferentiated connective tissue disease or early systemic lupus erythematosus was made, At the time being, he had been admitted to the hospital for blood transfusion and he suddenly developed jaundice, lower limbs edema, abdominal distention, and urine retention. His blood tests showed high urea level $240 \mathrm{mg} /$ $\mathrm{dl}$, serum creatinine $3.1 \mathrm{mg} / \mathrm{dl}$, high total bilirubin with elevated liver enzymes and low serum albumin. Suprapubic catheterization was done and urinary bladder was drained, ursodeoxycholic acid and liveril tablets were commenced. On the next day he developed sudden shortness of breath and his oxygen saturation has decreased; consequently, he was admitted to the intensive care unit and non-invasive ventilation was administered (CPAP), the patient also received methypredisolone $500 \mathrm{mg}$ for three days, for which he showed good response; however his condition deteriorated rapidly within hours and he developed respiratory failure. A possibility of COVID 19 was made; and a nasopharyngeal swab and throat swab were taken.

An endotracheal intubation was done and the patient died after few hours. The real time PT-PCR result was conclusive to the diagnosis of COVID 19 in the next day. AIHA is a destruction of red cells by autoantibodies, while many cases are idiopathic, others are associated with autoimmune, certain drugs and malignancies. Here we are presenting the first autoimmune hemolytic anaemia associated complicated by COVID 19 in Sudan, despite autoimmune hemolytic anaemia is rarely complicated by infections, but the treatment with immunosuppression effect patient immunity and become more vulnerable to infection. The first reported case of COVID 19 in Sudan by the federal Ministry of health was on 12 March 2020, the infrastructure and the health system in Sudan is extremely fragile not only for COVID 19 infection but to a lot of epidemic diseases, as they been reported by the World health organization. Today on 31 May 2020 the accumulative number of all patients been reported by Sudan Ministry of health is 5026 cases with the mortality rate of $5.2 \%$ which is very high.

\section{Acknowledgment}

This study is been done as collaborative project Between Medical and Cancer Research Institute (MCRI), Nyala, Sudan. And Institute of molecular biology. The case was mange and followed By Dr Ziryab Imad Taha, Rheumatology Department, Haj Al-Safi Teaching Hospital, Khartoum, Sudan.

\section{Patient consent}

Written consent has been obtained.

\section{Conflicts of interest}

All authors declare there are no conflicts of interest.

\section{References}

1. Zheng R, Yu Xu, Weiqing Wang, Guang Ning (2020) Spatial transmission of COVID-19 via public and private transportation in China. Travel Med Infect Dis 34: 101626. 
2. Lei Z, Huijuan Cao, Yusheng Jie, Zhanlian Huang, Xiaoyan Guo, Junfeng Chen (2020) A cross-sectional comparison of epidemiological and clinical features of patients with coronavirus disease (COVID-19) in Wuhan and outside Wuhan, China. Travel Med Infect Dis 35: 101664.

ISSN: $2574-1241$

DOI: $10.26717 /$ BJSTR.2020.29.004807

Mohammed EAE, Ziryab IT. Biomed J Sci \& Tech Res

(c) (P) This work is licensed under Creative Commons Attribution 4.0 License

Submission Link: https://biomedres.us/submit-manuscript.php
3. Ribas RM, Paola Amaral de Campos, Cristiane Silveira de Brito (2020) Coronavirus Disease 2019 (COVID-19) and healthcare-associated infections: Emerging and future challenges for public health in Brazil. Travel Med Infect Dis 17: 101675



